PANORAMA

ISSN: 1909-7433

ISSN: 2145-308X

ednorman@poligran.edu.co

Politécnico Grancolombiano

Colombia

\title{
REFORMAS UNIVERSITARIAS ECUATORIANAS: EL ACCESO A LA EDUCACIÓN SUPERIOR
}

Latorre-Villacís, Vianca Mercedes

REFORMAS UNIVERSITARIAS ECUATORIANAS: EL ACCESO A LA EDUCACIÓN SUPERIOR

PANORAMA, vol. 14, núm. 27, 2020

Politécnico Grancolombiano, Colombia

Disponible en: http://www.redalyc.org/articulo.oa?id=343964051005

DOI: https://doi.org/10.15765/pnrm.v14i27.1524

https://journal.poligran.edu.co/index.php/panorama/about/submissions\#copyrightNotice

Esta obra está bajo una Licencia Creative Commons Atribución-NoComercial-SinDerivar 4.0 Internacional. 


\title{
REFORMAS UNIVERSITARIAS ECUATORIANAS: EL ACCESO A LA EDUCACIÓN SUPERIOR
}

\author{
ECUADORIAN UNIVERSITY REFORMS: ACCESS TO \\ HIGHER EDUCATION
}

REFORMAS UNIVERSITÁRIAS EQUATORIANAS: ACESSO AO ENSINO SUPERIOR

\author{
Vianca Mercedes Latorre-Villacís viancalatorre@yahoo.es \\ Universidad Autónoma Metropolitana, UAM., México
}

PANORAMA, vol. 14, núm. 27, 2020

Politécnico Grancolombiano, Colombia

Recepción: 09 Septiembre 2019 Aprobación: 07 Julio 2020

DOI: https://doi.org/10.15765/ pnrm.v14i27.1524

Redalyc: http://www.redalyc.org/ articulo.oa?id $=343964051005$

https://journal.poligran.edu.co/ index.php/panorama/about/ submissions\# copyrightNotice CC BY-NC-ND
Resumen: El presente artículo tiene como objetivo revisar los efectos que la política tiene sobre el sistema de ingreso a la educación superior de Ecuador. Como método se realizó un análisis estadístico y cualitativo de la metodología de investigación: documental, estadístico y de entrevistas, para elaborar una discusión y recomendaciones sobre las políticas públicas y el cumplimiento de los objetivos de 2010 a 2017. Los resultados muestran cómo estos cambios se manifiestan mediante las evaluaciones de las universidades y su categorización, la planificación de procesos de gestión de calidad en la enseñanza y la pertinencia de la oferta académica para garantizar que el sistema de ingreso de los postulantes sea de manera meritocrática. La política de educación superior ha provocado una situación paradójica en este sistema, tanto en el ámbito nacional como dentro de cada institución de educación superior.

Palabras clave: Educación superior, política de educación, Ecuador, acceso, reforma universitaria.

Abstract: This article aims to review the effects that the policy has on Ecuador's higher education entry system. The method used was a statistical and qualitative analysis of the research methodology: documentary, statistical and interview, to develop a discussion and recommendations on public policy and the fulfillment of the objectives from 2010 to 2017 . The results show how these changes are manifested through university evaluations and their categorization, the planning of quality management processes in teaching and the relevance of academic offerings to ensure that the system of admission of applicants is meritocratic. Higher education policy has led to a paradoxical situation in this system, both nationally and within each higher education institution.

Keywords: Higher education, education policy, Ecuador, access, university reform.

Resumo: Este artigo visa rever os efeitos que a política tem no sistema de entrada no ensino superior do Equador. O método utilizado foi uma análise estatística e qualitativa da metodologia de investigação: documental, estatística e entrevista, para desenvolver uma discussão e recomendações sobre políticas públicas e o cumprimento dos objectivos de 2010 a 2017. Os resultados mostram como estas mudanças se manifestam através de avaliações universitárias e da sua categorização, do planeamento de processos de gestão da qualidade no ensino e da relevância das ofertas académicas para assegurar que o sistema de admissão de candidatos é meritocrático. A política do ensino superior conduziu a uma situação paradoxal neste sistema, tanto a nível nacional como dentro de cada instituição de ensino superior.

Palavras-chave: Ensino superior, política de educação, Equador, acesso, reforma universitária. 


\section{INTRODUCCIÓN}

Los motivos que impulsan esta pesquisa se encuentran dados por las limitaciones que viven muchos aspirantes antes de ingresar a las universidades; es decir, restricciones como exámenes sin un claro objetivo de lo que evalúan (aptitudes o conocimientos), la ineficiencia del proceso sistemático de esos exámenes, déficit de cupos universitarios y un cuello de botella generado por eliminar universidades, al mismo tiempo que se modificó la forma de ingresar a este sistema. Por esto, se estudiaron tales factores que incidieron en el comportamiento de la matrícula durante el período de 2012 a la actualidad.

Dentro de la agenda política ecuatoriana y social surgió el debate sobre la calidad, con profundos cambios en las reformas universitarias, mediante la nueva Constitución de 2008 y la Ley Orgánica de Educación Superior (LOES), vigente desde el 12 de octubre de 2010 (Asamblea Nacional, 2010). Estas reformas emergen con fuerza con las políticas a las que fueron sujetas las instituciones de educación superior (IES). Dicho proceso llevó a la suspensión definitiva de 17 universidades de Ecuador y al establecimiento de un nuevo sistema regulatorio que permitiera la evaluación, acreditación y aseguramiento de la calidad luego del bachillerato, lo cual se planteó en la nueva LOES 2010 (CES, 2012).

Los objetivos de la política de la Secretaría de Educación Superior, Ciencia, Tecnología e Innovación (SENESCYT), se centraron en:

- Evaluar a las IES mediante un proceso de tres etapas (2009, 2012 y 2014).

- Promover la educación técnica y tecnológica para los fines pertinentes del cambio en la matriz productiva.

- Uniformar el criterio de entrada con un examen. La matrícula en este nivel de la región latinoamericana llega alrededor de 18 millones de estudiantes, con una Tasa Bruta de Escolarización Superior (TBES), próxima al 38\% (CEPAL, 2013).

Por tanto, el objetivo general de este trabajo consiste en analizar la política y las consecuencias que ha tenido en el sistema educativo superior, tanto la política de evaluación institucional y la política de acceso. Por lo tanto, se estudiarán los efectos de la evaluación sobre las IES. Por otro lado, se explicará la política de acceso desde 2012 hasta la actualidad, con base en tres ejes de análisis (meritocracia, transparencia y discriminación positiva), con el fin de observar qué efectos ha tenido en los postulantes (Barbón Pérez \& Fernández Pino, 2018; Vega, Medina, Saquicela, \& Espinoza, 2019).

\section{Problema de investigación}

Ecuador ha pasado por profundos cambios en la ES, a partir de la Constitución de 2008 y la LOES de 2010. La evaluación de desempeño institucional de las IES, realizada por el Consejo Nacional de Evaluación y Acreditación (CONEA), entre junio y octubre de 2009, ha tratado de orientarse a generar los elementos para la construcción de una política 
más eficaz en la educación superior. Todo esto con el fin de depurar y mejorarla. Los hechos históricos empezaron con la evaluación de la universidad ecuatoriana en 2012, que realizó el actual Consejo de Evaluación, Acreditación y Aseguramiento de la Calidad de la Educación Superior del Ecuador (CEAACES).

Por ende, el presente trabajo inicia por una introducción al sistema de universidades en Ecuador, de acuerdo con el número de carreras que ofrecen -2.804 en 2017-, para adentrarse en el problema de investigación que enmarca la agenda política y social, que, además de debatir la calidad de la educación superior, se enfrentó a una mayor demanda de ES que la oferta existente, debido a que se puso a prueba[1] la capacidad del Estado y del gobierno de garantizar la continuidad de estudios de calidad con una demanda de más de 40 mil estudiantes, mediante un plan de contingencia que le costó al Estado USD \$3.497.718 (SENESCYT, 2015). De manera que las estadísticas para 2011 mostraban que existieron 11.196 inscritos para rendir el Examen Nacional de Educación Superior, mientras que para 2017 hubo 292.728 inscritos para rendir dicho examen.

Con respecto a la oferta, había 111.422 cupos en 2011, pero 123.154 para 2017. Es decir, el aumento fue de 281.532 postulantes[2] frente a 11.732 cupos[3] (Ramírez, 2018). De acuerdo con lo demostrado, se tuvo que establecer un nuevo sistema regulatorio con procesos de evaluación, acreditación y aseguramiento de la calidad.

La política gubernamental promovió la segmentación de las IES; adicionalmente, se dio la creación de institutos técnicos y tecnológicos (ITT), para suplir la eliminación de universidades y escuelas politécnicas. En 2008 se ofrecían 3.309 carreras, de las cuales 277 eran técnicas y tecnológicas. En 2015 se regularizaron 2.012 carreras de los ITT públicos. Y en 2017 se ofrecían 2.804 carreras, donde solamente 1.060 eran carreras de los ITT públicos (Ramírez, 2018). Por lo tanto, es notable la urgente necesidad de equilibrar la demanda de enseñanza superior con oferta de ITT, cuando de las 17 universidades cerradas en el período de clausuras 2012-2014 el 100\% fueron privadas.

Cabe recalcar que la ES está dividida en universidades y escuelas politécnicas, subdivididas por financiamiento y por oferta académica, más la reciente creación de ciertos institutos superiores para aumentar la oferta académica[4] y reconfigurar la matrícula de la educación superior (SIGMA, 2015). En este contexto de fuerte segmentación social, la educación superior se convierte en una herramienta privilegiada de búsqueda de una mejor inserción en el espectro social, a la vez que parece poder garantizar mayores niveles de cohesión e integración en una sociedad profundamente fragmentada. La educación superior está siendo fragmentada por estratos socioeconómicos[5] y por tipos de IES.

Según los tipos de IES, se ha categorizado a las universidades por las letras A, B, C y D y otros para jerarquizarlas de acuerdo con su calidad, como se visualiza en la tabla a continuación. Ello incide en los procesos meritocráticos de ingreso a las universidades, y puede llegar a responsabilizar al estudiante por sus orígenes sociales y excluir a la población, ya que no ingresó a una universidad mejor. 


\begin{tabular}{|c|c|c|c|}
\hline CATEGORÍA A & CATEGORÍA B & CATEGORIAA C & CATEGORÍA D \\
\hline Escuela Politécnica & Escuela Superior Politécnica de Chimborazo & Escuela Superior Politécnica & Universidad Agraria del Ecuador \\
\hline Nacional & Pontificia Universidad Católica del Ecuador & Agropecuaria de Manabi & Universidad Laica Eloy Alfaro de \\
\hline Escuela Superior & Universidad Casa Grande & Universidad de Especialidades & Manabí \\
\hline Politécnica del Litoral & Universidad Católica de Santiago de & 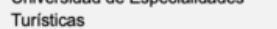 & Universidad Técnica Luis \\
\hline Universidad San & Guayaquil & Universidad del Pacifico Escuela & Vargas Torres de Esmeraldas \\
\hline Francisco de Quito & Universidad Central del Ecuador & de Negocios & Universidad Católica de Cuenca \\
\hline Universidad de Cuenca & Universidad del Azuay & Universidad Estatal de Bolivar & \\
\hline Universidad de las & Universidad Estatal de Milagro & Universidad Laica Vicente & \\
\hline Fuerzas Armadas & Universidad Nacional de Loja & Rocafuerte de Guayaquil & \\
\hline (ESPE) & Universidad Particular Internacional SEK & Universidad Metropolitana & \\
\hline Universidad de & Universidad Politécnica Salesiana & Universidad Nacional del & \\
\hline Especialidades Espiritu & Universidad Técnica de Ambato & Chimborazo & \\
\hline \multirow[t]{4}{*}{ Santo } & Universidad Técnica del Norte & Universidad Regional Autónoma & \\
\hline & Universidad Técnica Estatal de Quevedo & de los Andes & \\
\hline & Universidad Técnica Particular de Loja & Universidad Técnica de Babahoyo & \\
\hline & Universidad Tecnológica Empresarial de Gye & Universidad Tecnológica Israel & \\
\hline UNIVERSIDADES DE & Universidad Tecnologica Equinoccial & Universidad Estatal Peninsula de & \\
\hline POSTGRADO & Universidad Tecnológica Indoamérica & Santa Elena & \\
\hline Facultad & Universidad de los Hemisferios & Universidad Particular San & \\
\hline Latinoamericana de & Universidad Estatal Amazónica & Gregorio de Portoviejo & \\
\hline Ciencias Sociales & Universidad Politécnica del Carchi & Universidad Tecnológica & \\
\hline Universidad Andina & Universidad Iberoamericana & ECOTEC & \\
\hline \multirow[t]{8}{*}{ Simón Bolivar } & Universidad Técnica de Manabí & Universidad Técnica de Cotopaxi & \\
\hline & Universidad de las Américas & Universidad Estatal del Sur de & \\
\hline & Universidad Internacional del Ecuador & Manabí & \\
\hline & Universidad de Guayaquil & Universidad de Otavalo & \\
\hline & Universidad Técnica de Machala & & \\
\hline & UNIVERSIDADES DE POSGRADO & & \\
\hline & Instituto de Altos Estudios Nacionales & & \\
\hline & Universidades Públicas del Ecuador & & \\
\hline
\end{tabular}

Tabla 1.

Universidades, Escuelas Politécnicas, Institutos Técnicos

y Tecnológicos más demandados y Categorías (2017)

Fuente: SENESCYT (2017). Elaboración propia.

\section{Problema público}

En el marco[6] del Buen Vivir[7] (BV), la educación es considerada un bien público y gratuito, además de ser un área prioritaria y estratégica de desarrollo. Por tal razón, en la búsqueda de garantizar un acceso en igualdad de condiciones, la SENESCYT, por medio del Sistema Nacional de Nivelación y Admisión (SNNA), ejecuta la política pública que regula el ingreso a las IES públicas, bajo los principios de igualdad de oportunidades, meritocracia y transparencia (PNBV, 2013).

Un problema fundamental que merece una mayor concentración de política educativa es el acceso a las IES. De acuerdo con Ferreyra, Avitabile, Botero, Haimovich y Urzúa (2017), hay un aumento de estudiantes de tercer nivel en América Latina, pero esta situación alerta sobre las dificultades que ellos tienen para graduarse; es así como la tasa de cobertura pasó de $21 \%$ en 2000 al $43 \%$ en 2013 en dicha región. El incremento equivale a más de 20 millones de estudiantes que en la actualidad asisten a una de las 10.000 IES que funcionan en América Latina y el Caribe. Respecto a las universidades ecuatorianas, el exsecretario de SENESCYT, René Ramírez, remarcó que la matrícula creció 13 puntos porcentuales desde 2006, pues en la actualidad hay más de 303.000 nuevos estudiantes.

Es por esto por lo que el problema público de esta investigación es encontrar el mejor sistema de selección posible para acceder a las IES. La definición de "mejor" consiste en equilibrar los elementos o factores que más se acercan al sistema de selección más justo; es decir, un sistema 
no tan limitado y que considere equilibrar factores de meritocracia, transparencia y discriminación positiva.

El cambio o reconfiguración de la matrícula se ve influido por criterios de valor como la meritocracia, la transparencia y la discriminación positiva. En este contexto, la principal pregunta del presente trabajo puede definirse de la siguiente manera: ¿Cómo incidieron las reformas de 2010 en el sistema de admisión y nivelación ecuatorianos para el ingreso a la educación superior? Al tomar en consideración los indicadores meritocracia y calidad con las variables reformas y acceso a la educación superior se puede notar la contradicción de clausurar universidades de garaje, al mismo tiempo que se modificó la forma de ingresar a la educación superior; por tanto, la hipótesis de la presente investigación es: las reformas de 2010 no han sido suficientes para permitir una selección de aspirantes más justa, debido a que enfatizan el enfoque meritocrático, por encima de otros elementos como la discriminación positiva y el desarrollo de capacidades en contextos de exclusión y transparencia.

\section{MÉTODO}

Se realizó un análisis estadístico y cualitativo de la metodología de investigación: documental, estadístico y de entrevistas, para elaborar una discusión y recomendaciones sobre las políticas públicas y el cumplimiento de los objetivos de 2010 a 2017.

Las estrategias de investigación para este trabajo consistieron en el empleo del análisis documental, recopilación bibliográfica y estadística, con lecturas fundamentales de rendiciones de cuentas sobre la educación superior. Se llevaron a cabo entrevistas a expertos en la revolución por medio del conocimiento y el talento humano; es decir, funcionarios implicados en el proceso de las reformas al acceso y personas involucradas indirectamente o que recibieron las consecuencias de los primeros cambios institucionales. Ello permite confrontar al sistema de selección y complementar los datos estadísticos con el trabajo cualitativo de campo, por medio de entrevistas.

Se analizaron los recientes trabajos y documentos publicados en revistas especializadas, libros y conferencias realizados por organismos institucionales regionales. Además, se describirá de forma directa todas aquellas fuentes de los mismos actores internacionales como la Organización de las Naciones Unidas para la Educación, la Ciencia y la Cultura (UNESCO), y el Instituto Internacional de la UNESCO para la Educación Superior en América Latina y el Caribe (IESALC). Considerando las características del tema de estudio, el enfoque tiene una metodología multidisciplinar. Por una parte, se emplea un cuadro de las variables educación superior y acceso, como se aprecia en la tabla a continuación. También se desarrolló un enfoque metodológico descriptivo, complementándose con las técnicas estadísticas de las bases de datos más importantes. 

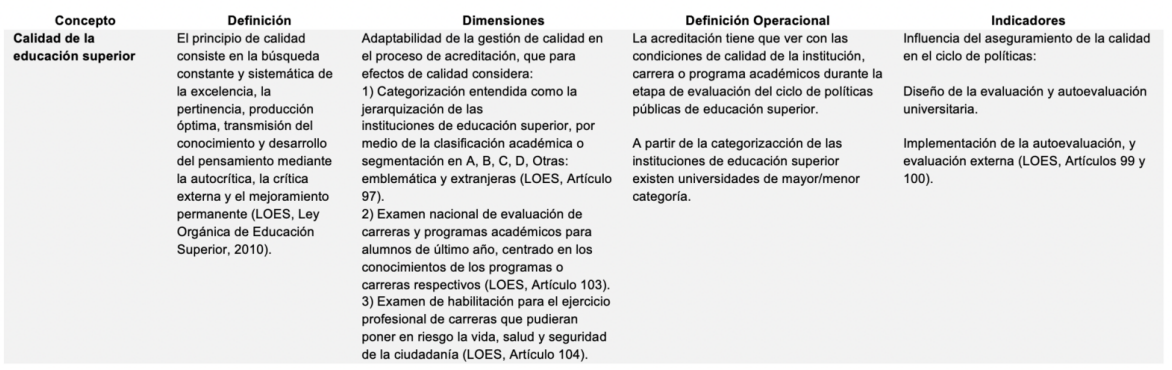

Tabla 2.

Operacionalización de las variables en educación superior

Fuente: LOES (2012). Elaboración propia.
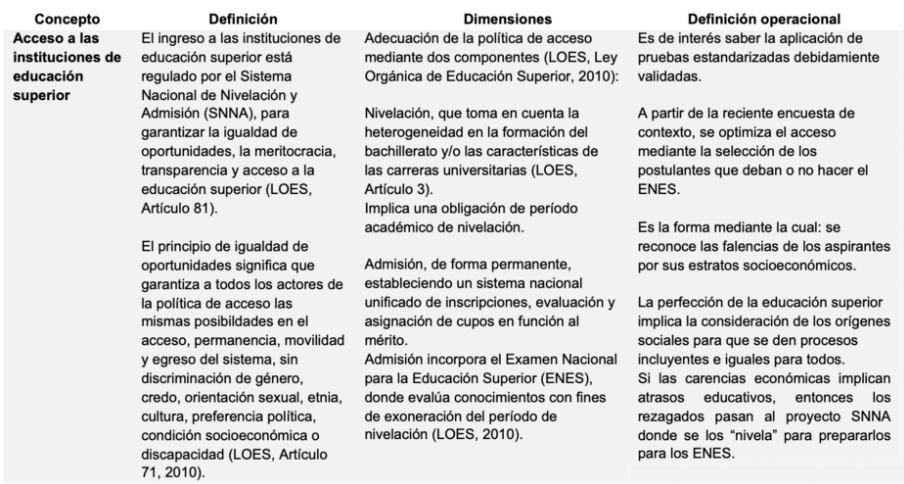

Tabla 3.

Operacionalización del acceso a la educación superior Fuente: SNNA (2014). Elaboración propia.

En el análisis no se incluyó la pauta que han marcado los organismos internacionales para todos los países latinoamericanos. Con base en las directrices de diversos organismos internacionales que se ocupan del tema educativo, la política de educación superior se inserta en un marco institucional de corte gerencial, que introduce nuevas reglas de operación e instrumentos de eficacia y eficiencia en el aparato político administrativo (Roqueñí, 2017). Además, los países ajustan sus políticas; por ende, la extensión, alcance e intención varían de uno a otro, en dependencia del sistema de educación superior existente y de la política educativa que el gobierno mantiene como prioridad. Ecuador tiene una política educativa con base en el Buen Vivir, como se ahondará en el capítulo primero.

\section{RESULTADOS}

A pesar de las reformas introducidas en el ámbito educativo superior ecuatoriano, y de haber sido considerado un área propicia para lograr aumentar la matrícula, en la práctica, las IES eliminadas, las pérdidas de carreras de diferentes ramas y la limitación de los aspirantes para ingresar al sistema han corroborado el insuficiente rediseño de la política institucional superior para lograr el acceso de cualquier individuo a la educación superior. 
Para explicar esta particular coyuntura, a lo largo de la investigación se realizaron un conjunto de planteamientos que permitieron desarrollar los objetivos propuestos en el trabajo. En pocas palabras, se estableció un marco teórico, se problematizó la temática, y ello permitió aclarar el enfoque de política educativa en Ecuador.

Igualmente, y con el objetivo de determinar la viabilidad de la propuesta de política equitativa, se realizó una comparación entre los sistemas de educación de los seis últimos años, cuyo análisis demostró las potencialidades, fortalezas y ventajas que se podrían alcanzar en materia del acceso, esto gracias a que el conjunto de capacidades instaladas de cada una de las IES de diferentes categorías de la A-D permitiría un mayor aprovechamiento de tales recursos, oferta, infraestructura y especialmente de docencia académica, al tiempo que se promovería la demanda de educación universitaria a nivel regional y en ciudades con mayor número de instituciones cotizadas, y se generaría el juego de la política meritocrática apoyada por un sistema transparente y una política de cuotas favorecedora del criterio de discriminación positiva.

Más adelante se estudiaron los criterios de valor que son meritocracia, transparencia y discriminación positiva de tres sistemas previos a la conclusión de que el último sistema de selección es el más adecuado, siempre y cuando se lleven a cabo las recomendaciones presentadas en este trabajo. La meritocracia, sin ser parte del agrado de toda la población beneficiaria, logró la segregación de quienes obtienen cupos de los que no. Lo que demostró que, a pesar de la retórica del más apto para acceder a una plaza, ha tenido más peso el cuello de botella generado por la eliminación de IES y la falta de capacidad de las universidades existentes en dar suficiente número de licenciaturas entre las del Estado, la SNNA e INEVAL, mediante los desacuerdos y negociaciones pendientes por intervenir en la nueva problemática por resolver: menos cupos para los aspirantes.

A pesar de las condiciones para favorecer la calidad a las que se sometieron todas las IES mediante la intervención que sufrieron para luego categorizarlas y/o re-categorizarlas en alguna jerarquía de mayor a menor calidad, se presentan signos de estancamiento. Esto debido a las falencias descubiertas a lo largo del trabajo de campo con las entrevistas a informantes claves del proceso de reformas institucionales. También, a las constantes divergencias entre las IES, ya que no hubo un previo proceso de contextualización de su situación como entidades públicas, tanto en lo político como en su autonomía, en lo cual la SENESCYT ha perdido el horizonte de cambiar las instituciones más que cortar de raíz a las que no cumplían con requisitos y parámetros alcanzables por todas las instituciones.

Además, se consideraron las debilidades y desventajas fácticas que hasta ahora han surgido de dichas reformas institucionales para la variable meritocracia, con respecto a las formas de ingreso. Por esto se determinó que, a pesar de los enormes beneficios políticos en la decisión por poner una categoría, la no existencia de una coordinación entre el sistema y las universidades, escuelas politécnicas e instituciones técnicas y tecnológicas 
permea la posibilidad de obtener la meta final de ofrecer una universidad pública equitativa para todos. De esta manera, la política de evaluación ha tenido eficacia, ya que se han cumplido sus objetivos, presentados en la introducción del presente trabajo, y lo ha logrado porque se clasificó y algunas IES han fomentado los criterios jurídicos-administrativos (academia, investigación, organización e infraestructura).

Sin embargo, en el caso de los docentes que son jubilados forzosamente por cumplir la edad de 65 años, es necesario destacar que no por ser mayor, necesariamente se es senil. De hecho, se argumenta que se está perdiendo a personas muy brillantes, que solo por la edad tienen que retirarse de la academia.

Por otro lado, se lograron los criterios de la política de acceso, de acuerdo con la meritocracia, la transparencia y la discriminación positiva, pero el argumento se basa en la crítica hacia la falta de capacidad instalada de las IES y los estudiantes rezagados para este sistema.

La propuesta de política pública inicia con la mejora de la variable calidad de las IES, lo cual desencadena la segunda propuesta que consiste en la política de equidad en el acceso mediante modificaciones al rediseño de los criterios de valor (meritocracia, transparencia y discriminación positiva). Para esto es necesario que se dé un proceso de universalización previo a la evaluación de los institutos y revisar los equipos y laboratorios construidos o faltantes dentro de la enseñanza superior.

Una crítica importante a la política de evaluación consiste en la drástica decisión de eliminar IES. Lo que hace falta es el establecimiento de una cuota para aquellos estratos institucionales no tan equipados, y así aumentar el criterio social de la contextualización. Esta es una solución, pero no es tan académica.

Para el caso de la política de acceso, cuando la meritocracia se sumerge en el discurso igualitario pierde por completo la perspectiva de los contextos y la diferencia de condiciones, ya que, si la igualdad es dar a todos lo mismo, la equidad es la justicia en todas las situaciones. Por tanto, en un Estado del Bienestar Inversor es necesaria la equidad para conseguir la efectividad de igualdad de oportunidades. De forma consecuente, una de las primeras conclusiones es que se prioricen la preparación de todos los individuos por igual desde el bachillerato general unificado (BGU), conocido también como educación media superior.

Se revela el problema de si debería retrasar o volver el tema de meritocracia hacia el ámbito laboral, ya que entra en debate el hecho que un sistema de selección evalúa la capacidad del individuo sobre si puede o no alcanzar un puntaje para recién ingresar a la enseñanza superior, mientras que el resto de proceso con sus ventajas y limitaciones podría simplemente demostrar que la persona es capaz de pasar un examen de muchos que vendrán en el futuro si es que obtiene un cupo para estudiar. Sin embargo, todo cambio crea resistencia, y trabajos de investigación como el presente se involucran en aquellos movimientos sociales emergentes cuyo fin es defender esas tendencias radicales de limitar o hacer más engorroso el acceso a la educación superior. 
A pesar de que el ingreso está mediado por el sistema de educación superior y las IES, no es suficiente que un examen sea el mecanismo indispensable y legítimo para insertarse en el sistema. Los entrevistados coinciden en que hay un problema estructural en la enseñanza superior.

Para comprobar la hipótesis, se reitera la crítica al sistema de selección, puesto que no ha sido suficiente democratizar el acceso a la educación superior de forma inclusiva o incluyente, ya que existen muchos rezagados que deciden desertar de este sistema mucho antes de rendir la prueba de educación superior, sumando el hecho de que la población que lo intenta proviene de diferentes clases sociales. De esta manera, las desigualdades individuales de cada postulante se terminan transformando en diferencias individuales, porque se quiere responsabilizar a los aspirantes por no ingresar a las IES, ya que no aprovecharon la igualdad de oportunidades que se les ofreció (Alban Taipe \& Mauricio Sánchez, 2018).

Además, el mérito intelectual puede abrir la universidad a cualquier persona en teoría, porque la riqueza, el capital cultural y las oportunidades están distribuidas desigualmente (Shea, 2010); es decir, la consecuencia del principio meritocrático favorece a los de clase media y alta, pero afecta a los grupos sociales con mayores desventajas (Barbón Pérez \& Fernández Pino, 2018; Jiménez Álvarez, Vega, Capa Mora, Fierro Jaramillo, \& Miguitama, 2019; Miranda-Calderón, Angulo-Hernández, \& RománLópez, 2018).

La estructura del sistema universal en la educación debería preocuparse por la igualdad de resultados, pues ahí se encuentra un problema de política pública muy específico; es decir, en vez de recalcar el tema de igualdad de oportunidades del sistema educativo, se debería hacer hincapié en que las condiciones de vida de cada individuo son distintas. Las estructuras universales se preocupan por generalizar solamente la igualdad de oportunidades, causando que no se prepare y contextualice al individuo postulante a la universidad, por ende, que no se tomen medidas discrecionales y la individualización. Además, se parte del supuesto que todos vienen con los mismos conocimientos y preparación y no se debería evaluar bajo los mismos parámetros a cada joven que desea acceder a las distintas IES (Jiménez Álvarez et al., 2019).

Entonces, la evaluación debe tomar en cuenta una política de cuotas más contundente que vele por el acceso de minorías sobre las mayorías a través de plazas apartadas antes de cada examen implementado. Es así como la igualdad de oportunidades no modifica las condiciones de desigualdad preexistentes con las que las personas acceden a los servicios de enseñanza superior. La postura de la presente investigación es que la propia idea de meritocracia es muy idealista, porque aprobar los exámenes previos depende de los estratos socioeconómicos de donde se provenga.

Con respecto a los sistemas de selección se concluye que las reformas al sistema de admisión y nivelación ecuatorianos para el ingreso a la educación superior desde 2010 han presentado aspectos positivos como la eliminación de IES de garaje y el aumento de la matrícula. Pero existen aspectos negativos como la frustración de los aspirantes que no consiguen cupos para carreras de tercer nivel aspiradas, limitado sistema por 
interponer un examen como mecanismo de medición discriminatoria de habilidades dentro de un contexto desigual de los aspirantes, insuficiente capacidad instalada de las IES y, por ende, poca oferta de cupos.

Fue una contradicción eliminar universidades de garaje al mismo tiempo que se modificó la forma de ingresar a la educación superior, porque se creó un "embudo", ya que de un lado el sistema intentaba aumentar la matrícula estudiantil y reconfigurarla; pero, por otro lado, disminuía el número de IES. Además, se le entrega una carga muy pesada a aquellas universidades que tienen ciertas carreras muy demandadas. Lo que habría que hacer es generar la misma carrera en otras universidades.

\section{DISCUSIÓN}

Las evaluaciones que se realicen en el futuro deberían ser voluntarias y propias de las IES para la mejora de su calidad, y así participar en la intervención, e impulsar la capacidad instalada de sus instituciones por medio de más ayuda del Estado con el consecuente incremento de los cupos universitarios.

Se requiere que la nivelación venga desde el bachillerato, previo a dar el examen para la educación superior, ya que las IES no se pueden responsabilizar por no traer resuelto el nivel de conocimientos y, por tanto, debe intervenir el papel del Estado por medio de evaluaciones a unidades educativas de enseñanza básica, secundaria y media superior, no con el fin de clausurarlas o eliminarlas, sino para llevar de la mano aquellos colegios privados y públicos que están por debajo de los estándares académicos.

Crear una estrategia para reabrir las IES que han sido clausuradas y generar los mecanismos para elevar la calidad de sus servicios. Es decir, no clausurar las universidades sino generar esquemas de cofinanciamiento para aquellas instituciones que no cubren los estándares de calidad establecidos, de tal manera que puedan recibir asistencia técnica, recursos humanos, entre otros elementos que fallen.

Por ejemplo, en aquellas IES que no tienen profesores, se podrían crear provisionalmente programas de estancias profesionales, ya que generaría el efecto indirecto sobre el empleo joven y que los egresados puedan trabajar provisionalmente como profesores.

Se recomienda hacer una encuesta a los egresados de bachillerato que planean transitar al sistema de educación superior, explorar las opciones que eligen entre las IES y las carreras profesionales que constituyen la oferta educativa a nivel superior para encontrar si estudian una carrera de su preferencia o primera opción, la influencia del nivel de conocimientos en la elección de IES privadas, la combinación de estudio y trabajo en caso de los que se encuentran en IES públicas.

El diseño de la política de evaluación institucional no consideró que al eliminar las IES iba a aumentar el déficit de cupos. Para la etapa de selección de carreras, el sistema podría discriminar entre dos opciones de carrera. Es decir, al escoger la opción de prioridad, el alumno podrá en la 
segunda postulación escoger una carrera relacionada a la de prioridad; por ejemplo, carreras jurisprudenciales.

Por otro lado, el incremento en el número de plazas en la educación superior no significa necesariamente ampliar el acceso a una gama más amplia de grupos sociales. El mérito intelectual depende del ingreso, capital cultural y oportunidades que son desigualmente distribuidos. Ecuador ha intentado ampliar el acceso sin cambiar su sistema de selección meritocrática; es decir, de 2012 a la actualidad existen muchos aspirantes que superan los puntajes referenciales, pero no consiguen entrar a las carreras que consideran de su prioridad.

La discusión que se ha llevado a cabo sobre la meritocracia consiste en que dicha variable segrega, ya que cuando se les insiste a los aspirantes en participar en este proceso, ellos sienten que se merecen lo que tienen, pero también piensan que los pobres se merecen su pobreza porque no han trabajado duro. Entonces, se merecen el sufrimiento que implica la pobreza. A pesar de que el sistema de acceso ecuatoriano se jacte de evaluar aptitudes y no conocimientos, todavía coexiste la creencia de que la élite triunfa en los exámenes y obtención de plazas porque es inteligente y trabajadora. Aquí entra en juego el papel de la etapa de nivelación, ya que necesita modificaciones a este proceso. Se propone que la nivelación se cambie de las IES a la educación media superior, pues, como se analizó en el capítulo III, en el caso de que el aspirante no pase el examen con un puntaje de 600/1.000, debe repetir el año escolar, por tanto, estas limitaciones se pueden mermar con unas modificaciones flexibles.

A lo largo de este trabajo se constató que las universidades públicas tienen un $10 \%$ de cupos para indígenas, afroecuatorianos, montuvios, ciudadanos con discapacidad y personas privadas de libertad; $5 \%$ de cupos para universidades particulares, y de $5 \%$ hasta $15 \%$ en universidades privadas. No obstante, se podría reservar el cupo para el siguiente semestre a las personas que sí sacaron el puntaje histórico necesario (puntajes extraordinarios). Además, se sugiere que se incremente a un mínimo de $50 \%$ la política de cuotas de grupos históricamente excluidos.

La superioridad y eficiencia de la enseñanza municipal y privada hace que los estudiantes cuenten con "aptitudes" o conocimientos mínimos necesarios para el ENES para el ingreso. Pero todos tienen oportunidad de entrar a una IES. El problema no es que no tengan esa ventana de oportunidad, sino que consiste en analizar a qué categoría de institución entran por quintiles socioeconómicos, por etnia y por género. Para futuras investigaciones, sería más interesante conocer a qué categorías de IES accedieron los grupos excluidos, ya que esto permitiría concluir si fue una inclusión igual o desigual.

Dentro de las entrevistas realizadas se pudo palpar un suceso inevitable; es decir, numéricamente habría suficientes cupos para enfrentar la demanda, pero cualitativamente no. Las universidades no solo deberían conformarse con ofertar los cupos de su potencial capacidad instalada, sino planificar incrementos en la infraestructura y talento humano para que la oferta pueda crecer. Además, aunque no haya vocación profesional, aunque haya déficit de cupos, el sistema debería preocuparse por mejorar 
la vocación profesional por medio de desplegar pestañas que indiquen carreras relacionadas con la primera prioridad.

La propuesta de política de acceso equitativo consiste en dos partes. Primero, el examen debe evaluar aptitudes y conocimientos siempre y cuando vaya de la mano con un incremento en las cuotas porcentuales raciales y socioeconómicas con prioridad en las universidades públicas. Igualmente, debe existir un apoyo a los que no se encuentran en estos grupos excluidos mediante la reserva de cupo, siempre y cuando alcancen los puntajes requeridos.

$\mathrm{Al}$ respecto de las desigualdades de género, el crecimiento cuantitativo de la participación femenina en la educación superior ha restado centralidad a esta problemática; en Ecuador, la presencia de la mujer se expresa por medio de una participación en los grupos de alto rendimiento (GAR), en los resultados del ENES de septiembre de 2015. La composición tuvo un $54 \%$ de mujeres y $46 \%$ de hombres. Esto no implicó, sin embargo, que la distribución de la población femenina en las áreas de conocimiento resulte pareja, sino que se concentra especialmente en Ciencias sociales y Humanidades.

Con respecto a la equidad, se argumenta que el sistema educativo debe buscar una combinación entre el mérito (evaluar aptitudes y conocimientos), y la acción afirmativa. Es decir, la política debe velar, aparte de los resultados de ingreso, porque los estudiantes de IES puedan mantenerse y culminar con éxito la carrera. También, estrechar las brechas entre las escuelas de menor calidad con las de mayor calidad para trabajar muy fuerte de la mano con aquellas IES que tienen baja calidad, para que de esta forma se dé una consideración de los orígenes (con las herramientas necesarias para mantenerse), y que no se los abandona. El reto es que el Estado ya ha definido sus carreras de prioridad. La forma de viabilizar esto consiste en la lucha social de los estudiantes por exigir sus carreras deseadas.

Finalmente, a lo largo de este trabajo se argumentó que la política de evaluación ha tenido efectos drásticos en la educación, ya que ha segmentado el sistema superior. Debido a esta jerarquización, existen aspirantes que ingresan a IES con calidad diferenciada de otras. Es decir, todos tienen cupo, pero no todas las instituciones tienen la misma calidad. Segundo, la política de acceso ha dejado fuera a muchos aspirantes, dado que se han eliminado IES y no se ha puesto atención a la creación de nuevas instituciones que no solo sean técnicas y tecnológicas.

En suma, la política de educación superior ha provocado una situación paradójica en el sistema de educación superior, tanto en el ámbito nacional como en el interior de cada IES: por un lado, el cierre de IES en un marco de restricciones presupuestarias hacia la autonomía de cada institución, tanto del sector público como del privado, que se han vuelto cada vez más elitistas debido a las limitaciones del cupo; por otro lado, un significativo aumento de la exigencia durante los exámenes de selección, acompañado de IES fuertemente segmentadas, por los distintos niveles de desarrollo académico que las caracterizan, y diferenciadas en categorías A, $\mathrm{B}, \mathrm{C}$ y D. Es decir, a pesar del acceso a institutos técnicos y tecnológicos 
de la población beneficiaria que no alcanza carreras de su prioridad, al propiciar enseñanza diferenciada de calidad se favorece una inclusión desigual y una persistencia de las desigualdades.

\section{REFERENCIAS}

Asamblea Nacional Ecuador. (2010). Ley Orgánica de Educación Superior, LOES. Recuperado de: https://www.educacionsuperior.gob.ec/wp-conte nt.

Alban Taipe, M. S., \& Mauricio Sánchez, D. (2018). Prediction of university dropout through technological factors: A case study in Ecuador. Espacios, 39(52). Retrieved from https://www.scopus.com/inward/record.uri?eid=2-s2.0-85058960 432\&partnerID $=40 \& \mathrm{md} 5=7 \mathrm{~d} 707 \mathrm{ccfecb} 33 \mathrm{cf1} 113 \mathrm{ef} 3 \mathrm{a} 20788696 \mathrm{f}$

Barbón Pérez, O. G., \& Fernández Pino, J. W. (2018). The role of strategic educational management in knowledge management, science, technology, and innovation in higher education . Educacion Medica, 19(1), 51-55. h ttps://doi.org/10.1016/j.edumed.2016.12.001

Comisión Económica para América Latina y El Caribe (CEPAL). (2013). Panorama social de América Latina. Santiago de Chile, Chile: CEPAL.

CES. (2012). Rendición de cuentas 2011-2012. Quito, Ecuador, pp. 19-20.

Ferreyra, M., Avitabile, C., Botero, J., Haimovich, F., \& Urzúa, S. (2017). Momento decisivo: la educación superior en América Latina y el Caribe. Washington, D.C.: Banco Mundial. Licencia: Creative Commons Attribution CC BY 3.0 IGO.

Jiménez Álvarez, L. S., Vega, N., Capa Mora, E. D., Fierro Jaramillo, N. C., \& Miguitama, P. Q. (2019). Learning teaching styles and strategies of University Students of Soil Science . Revista Electronica de Investigacion Educativa, 21(1). https://doi.org/10.24320/redie.2019.21.e04.1935

Miranda-Calderón, L. A., Angulo-Hernández, L. M., \& Román-López, G. (2018). The national university of Costa Rica program profiles, dynamics and challenges of the costa rican education: A proposal for pedagogical innovation and the production of materials and technological didactic resources. Revista Electronica Educare, 22(1). https://doi.org/10.15359/ ree.22-1.10

Ramírez, R. (2016). Universidad urgente para una sociedad emancipada. Quito, Ecuador: SENESCYT-IESALC.

Roqueñí, M. (2017). Expresiones de las desigualdades sociales en espacios universitarios asimétricos. Ciudad de México, México: Consejo Nacional de Ciencia y Tecnología (Conacyt).

SENPLADES. (2013a). Buen Vivir: Plan Nacional del Buen Vivir 2013-2017. Quito, Ecuador: SENPLADES.

SENESCYT. (2015b). Rendición de Cuentas. Quito, Ecuador: SENESCYT.

Shea, M. (2010). Desigualdad de acceso a la educación superior en el Reino Unido. En: Reencuentro. Ciudad de México, México: UAM-X. pp. 19-23.

SIGMA. (2015). Definición de la Oferta Educativa: Manual de Usuario. Recuperado de: https://www.uco.es $>$ atlas $>$ manualidoa

Vega, F., Medina, J., Saquicela, V., \& Espinoza, M. (2019). Towards an E-learning Platform Based on Interactive Visual Elements. 37th 
International Conference of the Chilean Computer Science Society, SCCC 2018, 2018-Novem. https://doi.org/10.1109/SCCC.2018.8705 246

[1] El 12 de abril de 2012, 14 universidades y escuelas politécnicas y sus 63 sedes fueron clausuradas o "suspendidas por falta de calidad académica". De 2007 a 2014 se cerraron 17 universidades y se crearon cinco nuevas: YachayTech, Ikiam, Universidad de las Artes y Universidad Nacional de Educación y la Universidad de Posgrado del Estado.

[2] El cálculo se obtuvo de restar la demanda de 2017 menos la de 2011; es decir, $292.728-11.196=281.532$ postulantes, el cual fue mayor a la oferta.

[3] El cálculo se obtuvo de restar la oferta de 2017 menos la de 2011; es decir, 123.154-111.422=11.732 cupos, el cual fue menor a la demanda.

[4] Por oferta académica se entiende la composición de los planes de estudio y de las asignaturas en disposición de ser impartidos por los docentes.

[5] Con estratos socioeconómicos se hace referencia al porcentaje de la población que es desfavorecida porque no ingresan a universidades de mejor categoría, lo cual genera una discusión por sus orígenes sociales, ya que muchos estudiantes no alcanzan los puntajes necesarios para las mejores IES y, como resultado, no se podría hablar de una democratización de la educación superior.

[6] La educación y el BV influyen de dos maneras: por un lado, el derecho a la educación es un elemento esencial del Buen Vivir, porque permite el desarrollo de las potencialidades humanas y, como tal, garantiza la igualdad de oportunidades para todos los individuos. Por otro lado, el BV es un eje esencial de la educación, en la medida en que el proceso educativo debe contemplar la preparación de futuros ciudadanos, con valores y conocimientos para fomentar el desarrollo del país.

[7] El Buen Vivir o Sumak Kawsay es alcanzar una vida de plena realización, reconociendo y respetando las diversidades, en armonía con nuestros semejantes y la naturaleza.

\section{Información adicional}

Para citar este articulo: / To cite this article: / Para citar este artigo: Latorre-Villacís, V. (2020). REFORMAS UNIVERSITARIAS ECUATORIANAS: EL ACCESO A LA EDUCACIÓN SUPERIOR. (P. Grancolombiano, Ed.). Revista Panorama, 14(27), DOI: http:// dx.doi.org/10.15765/pnrm.v14i27.1524 